\title{
Análisis del discurso
}

\author{
en \\ "Viaje a la Alcarria" \\ y "Nuevo viaje a la Alcarria" \\ de Camilo José Cela
}

Presentado por: Heba Alla Saad Zaghlol Abdul Hakim

Bajo la dirección de:

Prof. Dr. Abdul Kader Ateya Abu El-Enein.

Dra: Mona Salah El Din Shalan

Universidad de Ain Shams

Facultad de Al-Alsun

Departamento de Español

El Cairo -2012 


\section{INTRODUCCIÓN}

En este trabajo nos proponemos estudiar el discurso de dos obras de Camilo José Cela "Viaje a la Alcarria" y "Nuevo Viaje a la Alcarria" desde un enfoque sociolingǘstico, intentando reflejar la influencia de los rasgos geográficos y sociales sobre el lenguaje de la Alcarria ${ }^{1}$.

Una lengua no se habla igual por todos los hablantes, ni los mismos hablantes hablan o escriben igual en todas las situaciones comunicativas. Por ejemplo, el andaluz presenta muchas discrepancias frente al canario o al murciano, aunque todos son dialectos del castellano. Tampoco utilizan de la misma manera ese idioma las personas que viven en el medio rural, que las que viven en una ciudad. Ni siquiera, dos personas que viven en la misma casa hablan de la misma manera, seguramente el abuelo utiliza una variedad lingüística diferente de la del padre, y de las del nieto. Pero, es más, incluso una misma persona habla de manera muy diferente según en qué contexto se encuentre, no es lo mismo hablar con un amigo que con el director del colegio, o el jefe de la oficina. Todo ello da lugar a diferentes variedades dentro de una misma lengua. Como lo afirma Fernández Moreno (1998) en la cita siguiente:

La lengua es variable y se manifiesta de modo variable. [...] el hecho de la variación, definida como el uso alterno de formas diferentes de decir lo mismo, se puede encontrar prácticamente en todos los niveles

\footnotetext{
${ }^{1}$ Una comarca natural española que comprende la mayor parte del centro y del sur de la provincia de Guadalajara, el noroeste de la provincia de Cuenca (ambas en Castilla - La Mancha) y el sureste de la Comunidad de Madrid.
} 
de la lengua, desde el más concreto (fonético- fonológico) al más amplio (discurso, por ejemplo), pasando por la gramática y el léxico ${ }^{1}$.

Las "variedades de una lengua" son el resultado de factores geográficos, socioculturales y contextuales o situación comunicativa:

La sociolingüística centra una parte de sus preocupaciones en el estudio de la variación y de las variedades lingüísticas [...] una variedad lingüística es una manifestación del fenómeno llamado lenguaje que se define como un conjunto de elementos lingüísticos de similar distribución social. Dentro de esta definición, amplia donde las haya, quedan incluidas las lenguas de un hablante o de una comunidad de habla, los dialectos, los estilos, los registros, las jergas y cualquier otra manifestación lingüística, en la que se pueda observar un determinado uso o valor social ${ }^{2}$.

La procedencia geográfica del hablante, su origen social y la situación comunicativa dan lugar a la aparición de tres grupos de variedades lingüísticas. El primer grupo incluye las variedades geográficas, también llamadas diatópicas. Se trata, simplemente, de los dialectos:

De entre las variedades, una de las más conocidas es llamada dialecto, nombre que vamos a sustituir por variedad geográfica ${ }^{3}$.

\footnotetext{
${ }^{1}$ Francisco Fernández Moreno, Principios de sociolingüística y sociología del lenguaje, Ariel, Barcelona, 1998, p.19.

${ }^{2}$ Ibíd., p. 85.

${ }^{3}$ Karmele Rotaetxe Amusategi, Sociolingüística, Síntesis, Madrid, 1990, p. 22.
} 
Estas son las más obvias, y en las que todos pensamos cuando hablamos de diferencias lingüísticas. Como ya decimos, no habla igual un catalán que un gallego; un español que un argentino. A pesar de que todos ellos utilizan el mismo código y, sin ningún problema, podrían establecer una conversación entre ellos y se entendían perfectamente.

En el segundo grupo se encuentran las variedades sociales o diastráticas. Son los llamados sociolectos, los cuales identifican a los hablantes como miembros de un determinado grupo social. Aquí se incluyen los grupos cuya forma de hablar identificamos con la lengua vulgar, o las diferentes jergas juveniles, o la forma de hablar de grupos sociales marginales. De la misma manera, son sociolectos las variedades cultas, las utilizadas por doctores e investigadores universitarios en sus publicaciones, la jerga utilizada por los médicos, o la que usan los abogados. Las variedades sociales pueden reducirse a dos niveles extremos: nivel culto y nivel vulgar. El entorno de las variedades sociales contiene también lo que se llama lengua rural y urbana.

El tercer grupo abarca las variedades funcionales o diafásicas. Son los llamados registros lingüísticos. Estos registros aparecen en función de las características de la situación, o del contexto comunicativo en el que se encuentra el hablante. Por ejemplo, el registro coloquial lo utilizamos en situaciones informales, como entre familiares o amigos. En otras situaciones más formales nos esforzamos en utilizar el idioma con toda la corrección de la que somos capaces, como cuando hablamos con alguien importante, o nos encontramos en una situación oficial, o damos un discurso. Tampoco le hablamos igual a un niño que a un anciano. Todo ello implica contextos 
comunicativos distintos, y para cada contexto buscamos el registro más adecuado.

Los registros especializados han sido denominados también tecnolectos. Entre las variedades funcionales o diafásicas se encuentran además las jergas (variedad utilizada dentro de una profesión determinada) y los argots (variedad característica de un determinado grupo social: argot juvenil, argot del hampa, etc. $)^{1}$.

Garay Miguel (2006), en su tesis doctoral sobre la sociolingüística del discurso, explica que la variación sociolingüística viene como:

Consecuencia de la actuación de unos parámetros sociales-inherentes (sexo, edad y raza) y adquiridos (profesión, clase, nivel de instrucción) sobre niveles lingüísticos (fonéticos, morfológicos, léxicos, etc.). La clasificación de estas variaciones que da una lengua puede dar cuenta un hablante, junto a las derivadas de un uso plural de sistemas lingüísticos (bilingüismo, diglosia, pidgin, etc.) ${ }^{1}$.

En los apartados que siguen a continuación analizaremos los aspectos relacionados con las variedades funcionales o los registros diafásicos y las variedades sociales o diastráticas que aparecen en las dos obras estudiadas.

Diccionario de términos clave de ELE, publicado en
http://cvc.cervantes.es/enseñanza/biblioteca_ele/diccio_ele.




\section{LAS VARIEDADES DIAFÁSICOS}

\subsection{El registro coloquial}

En este apartado trataremos de destacar dos niveles de lengua utilizados por Cela en las dos obras que son: nivel popular y nivel vulgar. Algunos investigadores como F. Rodríguez Adrados (1992), define los niveles de habla como:

Diferencias no localizadas geográficamente, frente a las dialectales que son diferencias localizadas geográficamente ${ }^{2}$.

Y luego precisa que:

La noción de niveles de lengua está vinculada a la diferenciación social en clases o en grupos de diversos tipos ${ }^{3}$.

A continuación analizamos los recursos coloquiales que existen en las dos obras haciendo hincapié en el habla rural de la Alcarria y en el léxico utilizado por Cela ${ }^{4}$.

1 Miguel A. Garay, Sociolingüistica del discurso religioso, Universidad Nacional Mayor de San Marcos, Facultad de letras y ciencias humanas, LMA, Perú, 2006, p.3.

2 F. Rodríguez Adrados en José Alberto Miranda, Usos coloquiales del español, Publicaciones del Colegio de España, $1^{a}$ ed., Salamanca, 1992, p.22.

3 Ibídem.

${ }^{4}$ En esta parte de nuestro análisis hemos seguido la metodología empleada en el libro "Español coloquial- rasgos, formas y fraseología de la lengua diaria”, Eugenio Cascón Martín, 2a ed, Madrid, 2000. 
A continuación se detallan los recursos del registro coloquial utilizados por Cela que por una parte caracterizan al habla de la gente aldeana de la Alcarria y por otra parte marcan el estilo de Camilo José Cela.

\subsubsection{Las comparaciones}

El uso de las comparaciones se considera siempre como un recurso de extraordinaria eficacia en los grandes escritores:

Las comparaciones usadas por Cela a lo largo de su obra son abundantísimas y variadas en grado sumo. Pertenecen a dos grupos distintos: uno, el de comparaciones de su invención, marcadas por la personal garra del escritor; otro, el de las comparaciones de uso común, gastadas y tópicos, que podríamos estudiar en el habla familiar $^{1}$.

El uso de las comparaciones significa, simplemente el uso de un elemento (término iluminado) ${ }^{2}$ por medio de otro más conocido o concreto (término iluminante). Respecto a las dos obras de Cela podemos descubrir a través de los ejemplos que los términos iluminantes más frecuentes son por orden: los animales, los seres humanos, objetos, plantas y por último naturaleza inanimada.

\footnotetext{
${ }^{1}$ Sara Suárez, El léxico de Camilo José Cela, ed. Alfaguara, S.A., Barcelona, 1969, p.299.

2 Adoptamos la terminología de García Yerba en "Comparación épicas y realismo vital...”Arbor, LXI en Ibíd.
} 
En nuestra clasificación de las comparaciones, subrayamos en este apartado el iluminante, porque "siempre el iluminante es el más inspirado por la imaginación libre del escritor" ${ }^{* 1}$.

\subsubsection{Las comparaciones con animales}

Son los más abundantes en la obra de Cela en general y en sus libros de viajes en particular, donde el contacto con la naturaleza es continuo. Así surge una gran cantidad de comparaciones, en las cuales el término iluminante es del reino animal.

La relación entre los términos iluminado e iluminante se trata de determinadas cualidades que suelen aparecer en el término iluminado como se ve en los siguientes ejemplos:

- El hombre [...] tiene los dientes de color de tierra y grandes como los de los burros. P.32 - V. A. ${ }^{2}$

- ¡Jo, que el Camilo nada como un pez, que yo lo he visto! P.23 - V. A.

Es más frecuente en Cela comprar los dos términos a través de sus cualidades o circunstancias designadas generalmente por medio, de adjetivos, verbos o complementos que acompañan al iluminado. Este sistema de

1 - Ibíd., p.303.

${ }^{2}$ Utilizaremos las abreviaturas (V.A.) y (N.V.A.) respectivamente para aludir a Viaje a la Alcarria y Nuevo Viaje a la Alcarria a lo largo del presente trabajo. 
comparación se hace más concreto y restringido cuando el iluminante va acompañado también de clasificaciones y cualidades:

- El viajero deja volar libre la imaginación, que salta, como una torpe mariposa moribunda, rozando, en leves golpes, las paredes, los muebles. P.21 - V.A

- Sí, señor, se ponían como basiliscos, que era donde estaba la chispa. P.132 - N. V.A

En muchas ocasiones, la comparación cuenta con dos o más términos iluminantes. Este sistema "es una manifestación de la tendencia de Cela de acumulación"':

- Los gitanos igual que el jabalí y el gato garduño. P.23 - V. A

- Es un regalo esto de poder contemplar a dos mozas en su debido punto de sazón y sudando la gota gorda, esbeltas como gacelas y cabreadas como gatos. P.201 - N. V. A

Los iluminantes preferidos tanto en V. A como en N. V. A. son, casi siempre, pertenecen al léxico de animales domésticos y campesinos. Así, como hemos anotado antes (gatos, mulas, lirones, perros, burros, caballos, ratones, gacelas, etc.)

Sara Suárez en su libro sobre el léxico de Camilo José Cela subraya este fenómeno:

\footnotetext{
${ }^{1}$ Ibídem. 
Pero en Viaje a la Alcarria, otra vez en contacto con la naturaleza, la zoología vuelve a aportar cantidad y variedad de términos iluminantes, tomados de animales comunes, bien conocidos en sus características por toda clase de lectores ${ }^{1}$.

Son muchas las comparaciones que Cela usa de este tipo y que son verdaderamente populares:

Pero esto no aleja al autor de sus cauces normales: no hace más, por el contrario, que acercarlo a lo verdaderamente popular, pues el pueblo es especialmente aficionado a comparaciones recargadas y grotescas [...] Una vez más, el escritor se acerca a lo popular por el camino de la fantasía y funde su sistema constructivo con el del habla familiar. ${ }^{1}$

También la insistencia de usar lo popular y familiar se manifiesta en el incremento uso de comparaciones más tópicas y gastadas y que se encuentran más en su primer viaje a la Alcarria y se repiten varias veces, así por ejemplo:

¿Y de la madrina de guerra [...] que era más puta que las gallinas? P.39, El viajero, $[\ldots]$ se duerme como un lirón. P.157

\subsubsection{Comparaciones con seres humanos}

En la clasificación de las comparaciones más usadas vienen después de los animales. Los usa Cela tanto para hacer comparaciones con otros seres humanos como para animales, plantas o naturaleza inanimada.

\footnotetext{
${ }^{1}$ Ibíd., p.307. 
Sus elementos comparativos más preferidos son adjetivos que aluden a seres humanos. Por ejemplo:

- El pulso de la ciudad como el de un enfermo, late quedamente, como avergonzado de dejarse sentir. P.24- V.A

- El viajero entre la nuca de Oteliña y el rulé de las mozas del país, se siente como transportado a un paraíso. P.35 - N.V.A

En este tipo de comparación se nota que la gran parte se concentra en aludir a un iluminado (humano) con otro iluminante también (humano), especialmente en Nuevo V. A, donde Cela nos cita gran cantidad de apodos², tratando de explicarlos generalmente a través de otros más conocidos.

\subsubsection{Comparaciones con los objetos}

Respecto a las comparaciones cuyos términos iluminantes son objetivos, se puede suponerse que son inclasificables y amplísimas. Por ejemplo, se encuentra los que designan edificios, elementos de construcción, alimentos, mueble, puertas, trenes, etc...

- Los portales siguen cerrados, como las bolsas avaras. P.25 - V. A

- El viajero [...] piensa [...] mirar el alma de los caminantes asomándose a su mirada como el brocal de un pozo. P.26 - V. A

\footnotetext{
${ }^{1}$ Ibíd., p.314.

2 En las páginas que siguen vamos a estudiar los apodos que aparecen en los dos viajes como característica de lo coloquial en el habla de Alcarria.
} 


\subsubsection{Comparaciones con las plantas}

En cuanto a las comparaciones cuyos términos iluminantes son plantas son muy escasas y en general, los usa Cela para describir físicamente a sus personajes. La mayoría de las comparaciones de este tipo son tópicas y gastadas, así, por ejemplo: "gordos como manzanas", "colorada como una manzana" y otras comparaciones que se ven en las siguientes citas:

- A veces las casas de los vecinos ahogados, presumen de un balcón de geranios o de claveles rompedores, gordos, como manzanas. P.25 - V.A.

- Un pastorcito adolescente y una cabra pecan [...] a la sombra de un espino florecido de aromáticas florecitas blancas como la flor de azahar. P.70 V.A.

- Era como la madreselva que trepa alrededor del tronco del avellano. P.124 - N.V.A.

\subsubsection{Comparaciones con elementos de la naturaleza inanimada}

- El viajero [...] quisiera poder decir, al volver, las verdades de a puño que se explican, como el río que marcha, por sí solas. P.26 - V.A.

- Fuentes es como la tajamar del viento. P.55 - N.V.A.

\subsubsection{Otros tipos de comparaciones}

Junto a los tipos de comparación antes mencionados, hay otros menos usados, en su mayoría son gastadas y tópicas que representan el habla popular 
y familiar. Citamos como ejemplos los iluminantes religiosos y los que representan acciones:

- Aquella tabernita de en medio del campo era realmente algo muy parecido al paraíso terrenal. P.146 - V.A.

- Se acabó durmiendo como un bendito. P.146 - V.A.

- El hombre del tejar semejaba un angelito grandullón. P.119 - N.V.A.

Las comparaciones son una muestra del léxico popular preferido por Cela. Lo hace sin o con intención, como también concluye Sara Suárez:

Le deslumbran los iluminantes dislocados, absurdos y paradójicos propios del habla popular, y le encantan los gastados convertidos ya en tópico. A unos y a otros les da cabida con amor de su léxico y sobre sus moldes crea todo un mundo de comparaciones, unas veces elementales y sencillas, otras líricas o dramáticas, otras esperpénticas y grotescas ${ }^{1}$.

\subsubsection{Los diminutivos}

Cela es aficionado por los diminutivos, Carol Wasserman (1996) lo destaca en su estudio sobre la realidad e imaginación en los dos viajes de Cela:

Las palabras en diminutivo son tan abundantes en la lengua hablada que el autor-viajero las apunta con facilidad ${ }^{2}$

\footnotetext{
${ }^{1}$ Ibíd., p.337.

${ }^{2}$ Carol Wasserman, Realidad e imaginación; dos viajes a la Alcarria y la prosa de Camilo José Cela, Camineria hispánica: actas del II Congreso Internacional de Caminería 
Como se puede observar del análisis de los diminutivos que aparecen, respectivamente, en el primer viaje y en el segundo, los sufijos -ín, -ico, -uelo son los menos usados, mientras que -ito, illo son los más abundantes. Por ejemplo:

- Quien sabe si aún para comprarse una alfombrita...P.26 - V.A.

- El viajero busca una sombra para sentarse a descansar un rato, $[\ldots]$ comer un bocadito y tomar un pitillo. P.129 - V.A.

- ...detrás se apean un señor bajito y delgadito vestido con una cazadora muy brillante y cinco niños corrientes. P.236 - N.V.A.

El sufijo "ico" lo usa Cela muy pocas veces y lo aplica sobre todo, al mundo de la naturaleza y muy poco al ser humano. Se observa que la palabra que más se usa a través de su obra es vallecico.

- Por Ruguilla corre el agua en abundancia y en [...] el vallecico de Trasmuela hay más de mil plantas silvestres distintas. P.112- N.V.A

El sufijo "ín" de uso muy contario al anterior donde, se aplica con matiz despectivo, generalmente para personas y materiales poco importantes, por ejemplo:

- Con el cura y el viajero está un señorín muy acicalado. P. 206- N.V.A

Hispánica / coord. por Manuel Criado, Vol. 3, (Caminería literaria e hispanoaéricana), Manhattan Community College, Nueva York, 1996, p. 508. 
“ito" es el sufijo más abundante, tanto para sustantivos y adjetivos como para adverbios. En V. A hemos registrado unos 106 diminutivos. Entre los cuales 64 son con el sufijo ito, en N. V. A hemos recogido 88 diminutivo, de los cuales 55 con el sufijo ito. Esto quiere decir que el sufijo -ito representa casi el $80 \%$ de la totalidad de los diminutivos citados en las dos obras.

En general, y especialmente en el segundo V. A:

Deambulan numerosas referencias intertextuales al primer viaje a la Alcarria y hay, como en casi todos sus libros de viajes, una delectación en el detalle afectivo hacia los niños y los débiles en forma de diminutivos y sentimiento compasivo; hay un cuidado del elemento toponímico, de los mapas y la geografía, de la gastronomía, de la historia, del refranero como espejo de la sociedad tradicional o de los recursos naturales que esculpen el carácter de las gentes; nos habla largamente, por ejemplo, de las nueve clases de miel que se fabrican en la zona. ${ }^{1}$

Así, pues en el segundo viaje Cela estaba muy interesado de reflejar el tono tradicional de la gente del campo. Lo hacía con la ayuda del uso de los diminutivos, el refranero, el elemento toponímico, y otros mecanismos que vamos a citar a continuación. 


\subsubsection{Los apodos}

En la Alcarria de los cincuenta y de los ochenta cada uno tiene su apodo. La referencia de los apodos en el primer viaje de Cela volvió a aparecer en la segunda como un mecanismo muy frecuente y característico de su estilo:

Como en el primer viaje, vuelven a ser muy frecuentes las referencias a los motes que reciben los habitantes de los lugares que recorre. ${ }^{2}$

- A los de Azuqueca, les llaman cluecos. P.33- V.A.

Cela es amante de los apodos, como lo señala Carol Wasserman (1996):

El nombre y el apodo tienen una importancia enorme, como representativos de una persona o de una sociedad. Cela tiene, incluso, una obrita titulada "el coleccionista de apodos" donde dice que "los hombres somos malos, aunque nos creemos siempre mejores que el vecino"3.

En la Alcarria de los cincuenta cada vecino tiene su apodo, veremos en el párrafo siguiente una parte que se considera sin duda, reflejo de la herencia cultural de Brihuega, donde se enumera el personaje Julio Vacas en el primer viaje una cantidad de apodos conocidos del pueblo:

\footnotetext{
${ }^{1}$ José Luis Campal Fernández, Camilo José Cela, Viajero literario, Malabia: arte, cultura y sociedad, Barcelona, Montevideo, La Plata, abril 2007.

2 Ibídem.

3 Carol Wasserman, Realidad e imaginación; dos viajes a la Alcarria y la prosa de Camilo José Cela, Camineria hispánica: actas del II Congreso Internacional de Caminería 
- Mi nombre es Julio Vacas, aunque me llaman Portillo. En este pueblo cada hijo de vecino tiene su apodo, aquí no se libra nadie. Aquí tenemos un Capazorras, un Tamarón y un Quemado. Aquí hay un Chapitel, un Monafrita y un Cabezón, un Mahoma y un Padre Eterno, un Caldo y Agua y un Caracuesta, un Chil y Huevo y un Cabrito Ahumado, un Fraysevino, un Insurrecto, un Píoloco y un Mancobolo, un Taconeo, un Futiqui y un Pilatos; aquí señor mío, no nos privamos de nada. P.55

A. Zamora Vicente (2002) justifica el interés que tiene Cela por los apodos:

Preocupación por los nombres, por la voz exacta que despierte, a su conjuro, la hora, la sazón, la

trascendencia toda de la porcioncilla de esta tierra de Dios, donde penamos y sobrevivimos. Esto justifica la anhelante disposición de Camilo José Cela por recoger y usar el mayor número posible de apodos: Petaca, Tahonerito, Verduras, Tiriti, Baulero, Candil, Gangrena, Pegotechico, Túnel, Brazo fuerte, Chuletas, Gilillo, Cuerpo limpio, Chuchi, Cometa, Broncista, Fideísta, Panojista, Pelón, Ostioncito, Veneno chico, Fresquito de Valladolid, Lechuga... Preocupación viva, preocupación por el léxico. [...]En España -viejo país-, cada rincón tiene su nombre, no hay más que buscarlo.» Sí, hay que buscarlo. Nunca se agradecerá bastante a Camilo José Cela este

Hispánica / coord. por Manuel Criado, Vol. 3, (Caminería literaria e hispanoaéricana), Nueva York, 1996, p. 510. 
fervoroso esquilmo de la lengua, hecho con toda conciencia, luminosamente ${ }^{1}$.

Veremos a continuación una cantidad de apodos de Pastrana que nos cuenta don Fadrique en el segundo viaje:

- En el papel que don Fadrique dio al viajero venían apuntados los veintisiete apodos que siguen: Angelpatudo, Bailamisas, Cagacaga, Chimeneón, Docegorras, Espantabarracas, Fraysevino, Guitarrilla, Hombrebueno, Ingrávido, juanlanas, Kunfú, Laraña, lloralástimas, Mataborricos, Naricesdeamapol, Ñaña, Ojosdeaguamiel, Patapéndola, Quijotepijo, Roquegordo, Salivilla, Tuertopollero, Umbroso, Viruto, Yesero y Zurraco, hasta el final de 1a lista aún faltan muchos. P.242 N.V.A.

Se puede asegurar que para Cela el de los apodos no tiene nunca ni principio ni fin, esto es el cuento que nunca acabar ${ }^{-2}$.

\subsubsection{Léxico coloquial}

Son muy frecuentes en boca de los personajes de Cela el uso de ciertos sustantivos, adjetivos y verbos que en castellano presentan tantas acepciones, con lo familiar representan el pan de cada día en su obra, por ejemplo:

\footnotetext{
1 Alonso Zamora Vicente, Camilo José Cela, Acercamiento a un escritor, Alicante: Biblioteca Virtual Miguel de Cervantes, 2002, p.123.

2 - Camilo José Cela, Nuevo viaje a la Alcarria, p.242 
- El viajero, en una talabartería pequeñita [...] y que tiene un amo orondo [...] compra una testera de cuero. P.35- Col. Grueso o gordo.- V. A

- Se lo voy a regalar a un tío de mi señora, que es cura. P.35- Col. Sacerdote católico.- V. A

- El viajero piensa que quizá sea saludable su relativo hartazgo, porque tras la saturación, suele presentarse el arco iris del nirvana. P.15- Col. Estado de tranquilidad y serenidad grandes. N. V. A

- Esas cachas transeúntes, verbigracia, que semejan frutas en sazón del más remoto y mejor cuidado huerto del paraíso. P.18- Col. Ejemplo.- N. V. A

Algunas de estas palabras:

No siempre figuran en los diccionarios con tal matiz, aunque nos parezca increíble, dada su difusión en el habla llana de todas las clases sociales ${ }^{1}$.

Aunque, Sara Suárez observa que gran parte de los coloquialismos que se encuentran en las obras de Cela no figuran en los diccionarios, pero nos dedicamos solamente a la parte que aparece y figura en los diccionarios con la etiqueta $\mathrm{col}$. que significa coloquial ${ }^{2}$

Entre los sustantivos y adjetivos que recoja Cela es el uso de algunos para designar o calificar a la conducta moral de los personajes, describirlos

\footnotetext{
${ }^{1}$ Sara Suárez, El léxico de Camilo José Cela, ed. Alfaguara, S.A., Barcelona, 1969, p.46.

2 Debido a la dificultad que hemos encontrado en determinar de forma precisa las expresiones y palabras de uso coloquial y / o vulgar, hemos recurrido a la consulta de algunos diccionarios generales como el diccionario Clave o el DRAE.
} 
psicológicamente o físicamente, son términos peyorativos o compasivos de uso frecuente en el español familiar:

- Armando Mondéjar López es un niño preguntón. P.39- Col. Que pregunta con insistencia- V. A

- El pollo escucha con atención y hace gestos de asentimiento con la cabeza. P.103- Col. Muchacho joven- V. A

- ¡Lástima no reventases, perro! P.106- Col. Muy malo o indigno- V. A

- A las tiernas golfas del cabaret de las Llamas, con sus manos callosas y frías, sus pitillos al menudeo...P.21- Col. Prostituta.- N. V. A

- Cierta especialidad se encuentra en los usos de frases optativos o imperativos, locuciones oracionales que no son clasificables. Estos usos suelen incluirse en bloque dentro de la ambigua denominación de locuciones proverbiales o las locuciones interjectivas y en muy pocas veces los usa Cela para estos últimos signos de admiración. Todas presentan un uso puramente coloquial, como ejemplos citamos:

- Saca de la mochila los tomates, el pan y una lata de foie-gras, que se ha pasado de rosca. P.38- Col. Pasarse de rosca, excederse o ir más allá de lo debido.- V. A

- Las comparaciones no valen o valen poco pero, de cuando en cuando, se cuelan de rondón y sin avisar. P.21- Col. De repente o sin permiso.- N. V. A 


\subsubsection{Formulas de cortesía}

A Cela le encanta el uso de las formas de cortesía, que sin duda son muestra clara del uso coloquial de la lengua, asimismo, lo afirma Sara Suárez:

Los españoles son menos formalistas que otros pueblos en sus frases de cortesía, y lo son porque las dotan de más variedad, sin dejarlas llegar a la total fosilización, sino conservando las expresiones plenas originarias de empleo frecuente todavía, principalmente en el campo, pero también, al menos para ciertas situaciones, en las ciudades. ${ }^{1}$

Las formulas de cortesía son una muestra del habla familiar. Por otra parte, estas mismas son habituales del campo, citamos como ejemplos:

- Paco, para servir a Dios y a usted. P.34 - V.A.

- Adiós, que tenga usted suerte. P.41 - V.A.

- Gracias sean dadas a Dios. P.18 - N.V.A.

- Muchas gracias, señora, que Dios le conserve las mañas para la repostería y la buena disposición hacia los huérfanos, los nómadas y los desasistidos de la fortuna. P.53 - N.V.A.

\subsubsection{Interjecciones propias y impropias}

Como es bien sabido Cela se ocupa siempre por reproducir tan fielmente el habla natural y espontánea de los españoles, así que se concluye que en sus obras abundan las interjecciones y locuciones interjectivas.

\footnotetext{
${ }^{1}$ Sara Suárez, El léxico de Camilo José Cela, op.cit., p.192. 
En efecto, tanto los monólogos como las conversaciones de sus personajes están tan salpicados de estos términos, que reseñarlos todos sería imposible, y más aún con sus variadísimos matices ${ }^{1}$.

En sus dos obras encontramos las interjecciones propias e impropias, que suelen responder a un insistente deseo de Cela de reflejar el habla popular ${ }^{2}$.

La interjección jJo! donde quizás aparece más usada y con sus diferentes matices, también ;ay! Suele usarse mucho, tanto en V. A como en N. V. A, aunque era más en viaje a la Alcarria respondiendo al tono de melancolía y dolor del primer viaje y se considera:

Muy característica y propia de la lengua hablada. [...] mucho más coloquial para el dolor físico ${ }^{3}$.

Otras también que aparecen en sus formas simples y familiares como ¡caramba!, ¡caray!, ;huy! o jeh! Que se considera de uso más frecuente en los personajes de Cela.

Se usa más de una vez la interjección ¡Coño!, especialmente en N. V. A., y se considera:

\footnotetext{
1 Ibíd., p. 148

2 En los ejemplos que vamos a citar en los anexos, nos parece mejor citar las interjecciones más usadas sin pararnos en clasificar las propias e impropias, teniendo en cuenta que dentro de las llamadas impropias, las más usadas suelen reforzarse con ¡que!

${ }^{3}$ Sara Suárez, El léxico de Camilo José Cela, op.cit., 1969, p.151.
} 
La interjección malsonante de más uso en el español, ya olvidado totalmente $[\ldots]$ en Cela aparece sobre todo para ira, desagrado, mal humor ${ }^{1}$.

- ¡Coño, claro! Se llamaba Pilarín y las tragaba dobladas, ésa es la verdad. P.40 - N. V. A.

- ¡Coño!, qué comparación más original! P.236 - N. V. A.

- ¡Coño, Camilo! Me enteré que ibas a llegar y me dije: ¡De ésta no se me escapa! P.241 - N. V. A.

A veces se usa iPsche!, que se encuentra más en V. A para quitar importancia de lo que se dice o que va a decir o para contestar con ambigüedad como en el ejemplo siguiente:

- ¡Psche! Según como lo quiere mirar; no llevo prisa. P.63- V. A

Otra interjección que aparece una vez en V. A es ¡Zas! que designa acciones rápidas, sorprendentes o continuadas.

Según lo mencionado antes hemos visto la abundancia del registro coloquial - nivel popular y familiar que existe en las dos obras, no hay duda que hay otras manifestaciones de este nivel como puede ser:

- El que llamamos énfasis funcional, por ejemplo:

- Pues éramos muy amigos; ése y yo andábamos siempre juntos y

\footnotetext{
${ }^{1}$-Ibíd., p.154. 
el día que me dieron a mi el tiro también se lo dieron a él; fue en la sierra de Alcubierre, en Zaragoza. P.130- V. A.

- La exageración, por ejemplo:

- En la bodega de Luís Valles hay más vino del que pueda beberse una compañía de sedientos, en una vida entera y bien cumplida. P.225- N. V. A.

- Si este terreno fuera mío..., yo no descansaría nunca; casi ni para dormir. P.130- V. A.

\subsubsection{Las unidades fraseológicas}

Dentro de las características que sobresalen en Cela es la tendencia de reforzar el sentido usando refranes y sentencias en forma pintoresca y a veces grotesca, nos explica Sara Suárez (1969) esta afición en Cela:

Cela es aficionadísimo a todas estas manifestaciones de la sagaz y secular experiencia humana colectiva que en español ha cristalizado en formas particularmente pintorescas, graciosas y llenas de sabor popular, condiciones sobradas para que cuenten con todas las simpatías de nuestro autor; sobre todo en sus últimos libros, los refranes y las sentencias se han convertido en inagotable fuente de comicidad y desparpajo. ${ }^{1}$

\footnotetext{
${ }^{1}$ Sara Suárez, El léxico de Camilo José Cela, op. cit, Barcelona, 1969, p.221 
Cela (1963) mismo subraya:

Descubrimos, hace ya años, que hay refranes para todo ${ }^{1}$.

De acuerdo con esto Cela usa el refrán para expresar todo, y si no hay a veces los inventa. Por otro lado, el refrán se considera, junto con tantas otras manifestaciones, una parte heredada que refleja como piensa la gente en cada sociedad. A continuación, vamos a exponer algunos utilizados en la Alcarria de los cincuenta y de los ochenta y que aparecen en las obras analizadas:

- Cela no cita siempre el refrán, la locución o la sentencia completos, a veces una parte para hacer trabajar la mentalidad del personaje:

- Igual vuelven como Dios manda, que con más julepe que con una cuadrilla de cómicas. P.44- V. A

- Siete virtudes tiene la sopa: saca el hambre, sed enfada, y pone la cara colorada. P.50- N. V. A

En este párrafo y como explica García Marquina:

Las reflexiones de Martín están llenas de sabiduría popular y Cela recrea un lenguaje rural exacto y regocijante. Basta citar la muestra de la desconfianza sobre las chicas que van a servir a Madrid, algunas de las cuales vuelven con más julepe que una cuadrilla de cómicas. El campesino está dotado de un acervo de experiencias vitales, en parte heredadas y

\footnotetext{
${ }^{1}$ Camilo José Cela, Las compañas convenientes, $1^{\text {a }}$ ed., Noguer, Barcelona, 1963, p.226. 
codificadas en el refranero, las sentencias conocidas, el folclore y los dichos y coplas, y en parte experimentadas por sí mismas. ${ }^{1}$

- Más de una vez los refranes vienen uno tras otro continuamente tanto completos o incompletos:

- El viajero siempre tuvo muy claras tres cosas: que lo que abunda no daña, que nunca por mucho trigo es mal año y que más vale tener que desear. P.60- V. A.

- Al viajera le duele que su viaje esté dando las boqueadas, pero se conforma pensando dos cosas: que menos da una piedra y que no hay ni bien ni mal que cien años dure. P.219- N. V. A.

- Muchas veces se ve el uso de las unidades fraseológicas acompañado con una explicación u opinión previa en boca de los personajes:

- El niño ignora que las apariencias engañan, que debajo de una mala capa puede esconderse un buen bebedor, que en el peche del viajero, de extraño, quizá temeroso aspecto, encontraría un corazón de par en par abierto, como las puertas del campo. P. 27 - V.A.

- El refranero, a veces, no es justo y se deja llevar por la ruina malquerencia. De Alcocer ni mula ni mujer, ni hombre ni puede ser. Cosas muy parecidas se dicen de

\footnotetext{
${ }^{1}$ Francisco García Marquina, Guía viaje a la Alcarria, Aache, Madrid, 1993, p.73 
otros cien sitios, todo estriba en que la letanía pegue o no pegue. P.188 - N.V.A.

Sin embargo, creemos que lo más frecuente en Cela es recoger el refrán para aludir a los fenómenos atmosféricos o geográficos y las características de la sociedad en que vive la gente del campo y de la Alcarria en especial:

- Los ríos unen y las montañas separan, es la vieja sabiduría: no hay otra división que valga P.22- V.A.

- Dice el refrán: Tórtola de Henares está en la carretera que lleva a Hita y a Jadraque. P. 43 - V.A.

Entre los refranes que aluden a las características de los pueblos y la naturaleza geográfica del lugar citamos los siguientes:

- La pena fue que se perdieran los baños de Carlos III, que eran famosos de toda España. Ya sabrá usted lo que decía el refrán: que Trillo todo lo cura menos gálico y locura. P.88- V. A

- Caspueñas y Gajanejos, buena tierra de conejos, dice el refrán. P.64 - N.V.A.

- Yela está frente a Barriopedro y al otro lado, o sea detrás de Cívica, y medio escondido. Yela está en terreno muy frío a medio andar entre Masegoso y Brihuega. Hay un refrán que lo dice bien a las claras: entre Masegoso y Brihuega siempre Yela, que suena lo mismo que hiele del verbo helar. P.101 - N.V.A. 
La afición que tiene Cela por lo popular y vulgar lo lleva muchas veces a hacer un acopio de las formulas hechas, sentencias y refranes que expresan un concepto sobre la vida, el hombre, las cosas y se emplean para opinión, consejo, consuelo, disculpa, etc...a continuación vamos a ver algunos:

- Que de todo hay en la viña del señor. P.27 -V.A.

- Pues le voy a regalar a usted dos libros que tengo en mucha estima. Son muy antiguos, son dos libros de sabios. Por ellos no quiere nada: haz bien y no mires a quien. P. 57

- Que mañana como los pájaros del cielo, confían en que Dios proveerá. P.62

- Pero recuerde lo que decía Mateo Alemán: que no hay vasija que mide los gustos ni balanza que los iguale. P.18 - N.V.A.

- Hay un refrán que dice: riqueza vieja es la nobleza. P.30 - N.V.A.

Algunas veces aparecen las sentencias que hacen referencia a ciertas condiciones humanas o que aluden a los defectos y virtudes, por ejemplo:

- El que no espabila, ya sabe lo que le espera. P.82- V. A

Lo dijo el personaje don Estanislao de Kostka, sobre el cual Francisco García Marquina (1993) comenta:

$\mathrm{Su}$ discurso se articula sobre varios refranes y sentencias, que instituyen la desconfianza como base de cualquier relación: Los de Guadalajara no mantienen su palabra; nadie es de fiar, excepto el Papa; 
todo el que come es porque roba; en definitiva: El que no espabila, ya sabe lo que le espera. ${ }^{1}$

De igual modo Cela deja que sus personajes inventen las sentencias que como ayuda al personaje ayuda al narrador para reflejar cierta clase social:

- Si pasas por Armuña sin criticarte, pasas por el infierno sin condenarte. P.61 - V. A

- Uno para todos y todos para uno. P.67- V. A

- Por aquí se dice: lo que quieren los de Horche y los de Usano, no lo quieren los cristianos. P.228 - N. V. A

- ¡Más vale tarde que nunca! P.248 - N. V. A

Alonso Zamora Vicente (2002) dice afirmando el gusto que tiene Cela por los refranes:

Esta voluntad de estilo es la que nos explica el tono decididamente coloquial y rural (en el más noble sentido) de la lengua de Camilo José Cela. [...] . Léxico, sin embargo, insustituible en esta andadura intelectual. De ahí también la presencia de copiosos refranes y frasecillas hechas o proverbiales, la mayor parte incorporados a la narración, diluidos en el diálogo, como ha ocurrido siempre. [...]Contenido tradicional, personajes

1 Ibíd., p.137. 
de picaresca, regusto adormilado en la voz del pueblo, ¿no nos suena todo esto a los momentos más logrados de la literatura española? ${ }^{1}$

Cela mismo dice en su segunda viaje a la Alcarria:

- Don Fadrique tiene afición a coleccionar refranes, coplas, apodos y demás sabidurías populares, en esto coincide con el viajero. P.242

Se puede asegurar que el uso del refrán para Cela:

Ocurre, sobre todo, en los libros de viajes, y es una afición que va incrementándose en él. Ya en V. A y en El gallego y su cuadrillo aparecen algunos, especialmente refranes, que califican o caracterizan a un pueblo. ${ }^{2}$

En la parte anterior hemos visto, sobre todo, el léxico que Cela toma del idioma callejero, es decir, distintas manifestaciones del español hablado y vulgar. Cela mismo dijo en una entrevista publicada en Pueblo con fecha 7 de enero de 1967:

Todo lo que escribo y que parece inventado, está inventado. Son términos que se emplean. Lo que pasa es que han desaparecido de los diccionarios. Bueno... Allá quien haga los diccionarios. ${ }^{1}$

\footnotetext{
${ }^{1}$ Alonso Zamora Vicente, Camilo José Cela, Acercamiento a un escritor, Gredos, D.L. Madrid, 1962., p.125.

${ }^{2}$ Sara Suárez, El léxico de Camilo José Cela, op.cit., p.229.
} 


\section{LAS VARIEDADES DIASTRÁTICAS}

\subsection{El registro vulgar}

Los vulgarismos son muy abundantes en la obra de Cela, a causa de la gran cantidad de gente vulgar y callejera que aparecen en las páginas de sus obras. Así pues, no pocas veces utiliza el narrador un lenguaje de tono vulgar, "sobre todo madrileño"2.

Alonso Zamora Vicente (2002) afirma la misma idea:

A lo largo del libro solamente vamos a encontrarnos y a hablar con pueblo, con gentes humildes, modestas (pastorcillos, labriegos, posaderos, arrieros, buhoneros, vagabundos), la variopinta agrupación pasajera de un vagón de tercera ${ }^{3}$.

A. M ${ }^{\text {a. }}$ Vigara Tauste (1998) se refiere al término "vulgar" como:

Término inexcusablemente indicador de nivel y con connotaciones peyorativas $^{4}$.

Asimismo lo define Antonio Briz (1998) como sigue:

${ }^{1}$ Ibíd., p. 275.

${ }^{2}$ Sara Suárez, El léxico de Camilo José Cela, op. cit., p.49.

${ }^{3}$ Alonso Zamora Vicente, Camilo José Cela, Acercamiento a un escritor, op.cit., p. 93.

${ }^{4}$ Cito a través de Antonio Briz, El español coloquial: Situación y uso, Arco- libros, S.L. 2a ed., Madrid, 1998, p.26. 
Con el término vulgar nos referimos a ciertos usos incorrectos, anómalos o al margen de la norma estándar y de las normas regionales, resultantes de un nivel de lengua bajo. ${ }^{1}$

Lo vulgar igual que lo coloquial viene a consecuencia de rasgos sociales y no geográficos:

Lo vulgar no se localiza en una región determinada, no indica, por tanto, procedencia geográfica en los hablantes, sino una categoría social baja. [...] vulgarismos, expresiones que revelan un descuido y una falta de cultivo que se reflejan en todos los demás aspectos de la personalidad. ${ }^{2}$

Aunque, esto se contradice a la opinión de Vicente García De Diego de considerar el habla vulgar como un factor determinante del habla de la Alcarria que se debe a las perspectivas geolingüísticas que caracterizan la zona:

Este castellano vulgar en algunos elementos es coincidentes con elementos regionales próximos; fundamentalmente es el habla del pueblo rural y bajo de ambas Castillas ${ }^{3}$.

Pero, el problema que nos enfrenta en distinguir coloquiales de vulgarismos, es aunque:

\footnotetext{
1 Ibídem.

2 Manuel Muñoz Cortes, El español vulgar, Descripción de sus fenómenos y métodos de corrección, ed.cit, p.25.

3 Vicente García De Diego, Dialectología española, $3^{\mathrm{a}}$ ed., Cultura Hispánica, Madrid, 1978 , p.396.
} 
La Real Academia Española dé como familiares los madrileñismos chipén y napias, no cabe duda que aún son vulgares en el sentir de una parte de los hablantes, españoles ${ }^{1}$.

Dentro de los vulgarismos que se encuentran en las dos obras es la debilitación de la sonora intervocálica del sufijo ado reducido a -ao. Cela lo emplea, a veces en boca de sus personajes toscos:

- Que no se comprometida asta ver el resultao de ese chico. P.120 - V. A

- Biendose alguna vez precisao no tiene na mas que escribirme. P.121 V. A

- A los de Tendilla les dicen encenagaos, ya se sabe que esto va en gustos y también en ganas de marear. . P.221 - N. V. A

También se nota a veces el uso de éste y aquí para designar la persona presente, siempre ocurre entre la gente inculta, especialmente en Nuevo viaje a la Alcarria:

- Ya me dijo aquí que iba a venir usted. P.205

- Nada; éste salió algo grilla pero es buen muchacho, ahora se le murió la madre, bueno, va ya para tres años, y lo único que quiere es que no le peguen patadas y le hagan algo de caso, tampoco es pedir demasiado. P.226

\footnotetext{
1 Zamora Vicente, Lengua, literatura e intimidad en Sara Suárez, El léxico de Camilo José Cela, op. cit., p.50
} 
Es muy abundante el uso de un léxico lleno de tantos vocablos y giros totalmente vulgares:

- Un buey rubio y viejo, de largos cuernos y cara afilada, como un caballero toledano, bebe, no más que acariciando el agua con el morro cano, en el pilón de una fuente fecunda. P.53- Vulg. Labios. - V. A

- Un hombre mea gloriosamente, desafiadoramente, desde el balcón. P.114- Vulg. Reírse mucho - V. A

- Al viajero le gusta más el cachondo y albortador orrer de la vida. P.16- Vulg. Malson. Divertido, gracioso o burlón. - V. A

- Ahora están de moda los confusos travestidos en determinado de las diáfanas putas...P.22- Vulg. Prostituta - V. A

- Un motorista muy espectacular y aparatoso se mete con Oteliña y el viajero, por señas, se caga en su padre. P.29- Cagarse en algo Vulg. Maldecirlo. - N. V. A

Por otra parte, se encuentra ciertos falsos plurales de posibles raíces madrileñas y el uso del género femenino para designar masculino o viceversa:

- A mi me pasa los mismo... P.60 - V. A

- Dice los jardines en vez de jardín. P.63 - V. A

- ¡Dígalo, hombre, dígalo! Yo no soy un voceras. P.126 - Viaje a la Alcarria

- Al lado del fuego bajo, ya casi consumido, un hombre dormita y un gato duerme. P.137 - V. A 
- Por un callecita pasa $[\ldots]$ un burro en cueros, [...] enseña los dientes de la color de la calabaza. P.53 - N. V. A

- Fuentes es como la tajamar del viento. P.55 - N. V. A

- Falta poco para el mediodía y la calor empieza a picar en las túrdigas del organismo. P.59 - N. V. A

- En Alcobujate las coles. P.189 - N. V. A

El uso del género femenino para designar a masculino o viceversa, es también una de las características que define a Castilla la Nueva donde se encuentra Alcarria:

En el ámbito de la morfología nominal, nuestra región muestra también características que coinciden con las otras tierras. Llama la atención, sin embargo, el uso masculino de algunas voces femeninas ${ }^{1}$.

No muchas veces se mezcla lo vulgar con lo coloquial, por ejemplo, el artículo con un adjetivo como la expresión que se usa tanto en V. A como en N. V. A y que significa "por casualidad":

- Va usted a Zaragoza; por un casual. P.36 - V. A

- ¿Este Cimbre era de Sigüenza, por un casual? P.89- N. V. A

- ¿Este Cimbre no había sido sacristán en Sacecorbo, por un casual? P.90- N. V. A. 


\subsection{Localismos y expresiones rurales}

La lengua española tiene una variedad dentro de una unidad esencial. Muchas veces choca un empleo de palabras que a otro español le resultan desusadas.

En este sentido se puede asegurar que:

El lenguaje es una institución social de gran poder conservador y esta misma estabilidad es lo que le permite cumplir su misión comunicadora. Y correlativamente, todo cambio de estructura social se traduce en cambios de estructura lingüística. ${ }^{2}$

Cela en sus dos viajes a la Alcarria se concentró con la gente pobre. La vida del campo fue reflejada a través de la gente rural. Casi todos los personajes de las dos novelas son campesinos que utilizan, sobre todo, vocabulario aldeano, así también como lo afirma Francisco García Marquina (2002):

Pero la mayor fuente de información sobre la vida del campo es el propio lenguaje que emplean los aldeanos. Ya sabemos que Cela, en sus indignaciones, está más atento a los campesinos y la gente del pueblo que

\footnotetext{
${ }^{1}$ Manuel Alvaro, Manual de Dialectología hispánica, ed. Ariel S. A., Barcelona, 1999, p.223

${ }_{2}^{2}$ Francisco García Marquina, Guía del viaje a la Alcarria, op.cit. p.200.
} 
a los eruditos y académicos, por lo que sus observaciones son siembre muy esclarecedoras ${ }^{1}$.

Casi todos los críticos afirman la misma idea que V. A era la primera y más importante piedra sobre la que constituyó su diccionario secreto que contiene:

Motes personales, gentilicios, refranes y díceres del pueblo que Cela ha recogido durante más de 30 años ${ }^{2}$.

Según Manuel Seco, en 1950, en las páginas de V. A, Cela ya dio su primer síntoma de esa "sabia manía de cosechar los nombres burlescos" que lo ha llevado a comenzar esta obra. ${ }^{3}$

Por su parte, Francisco García Marquina (2002) analiza la razón de utilizar un vocabulario aldeano en las obras de viaje:

La inmovilidad secular del campo ha consagrado relictos de expresión que sorprende a los viajeros. Esto es comprobable a primera mano en lo referente al léxico, donde Cela encuentra y exhibe hallazgos de belleza fonética y sabor arcaico. Y podemos citar los que acaba de poner en boca del pastor Roque, artuña, adalid, soceto. Si Cela le hubiera pedido un cencerro como recuerdo, Roque le hubiera preguntado: ¿Quién usted un

\footnotetext{
${ }^{1}$ Ibidem.

${ }^{2}$ Cristina Aldaz, Resurge el Cela más "popular", El Mundo-Cultura, Jueves, 16 de abril de 1998.

${ }^{3}$ Ibíd.
} 
pito, un cascarro, una grilleta, una cencerrilla, una cencerra, un truco, un campanillo o una zumba?, con lo cual hubiera aumentado la admiración del escritor ${ }^{1}$.

Cela, pues en las dos novelas y sin duda alguna:

Aprovecha [...] el léxico dialectal, los regionalismos, y hasta los localismos, cuando le parecen expresivos y sonoros. Los recoge, sobre todo, en los libros de viaje ${ }^{2}$.

Por eso, no tenemos duda que su lenguaje era sobre todo, localista, miramos como también lo explica Sara Suárez (1996):

Sus últimos libros de viajes, que son, ciertamente, un riquísimo venero de lengua castiza, concreta y poco conocida (con frecuencia, muy localista), pero usada a veces en aluvión, de tal modo que la cantidad y el hacinamiento perjudican a la claridad y a la viveza de la narración ${ }^{3}$.

Dice Francisco García Marquina (2002) afirmando la idea de que Cela usa, sobre todo un dialecto alcarreño:

Mientras el viajero reposa y charla con los vecinos podremos hacer una ligera excursión lingüística sobre el léxico que Cela empleará en su viaje a la Alcarria y que procede del alcarreo que ha realizado en el camino

\footnotetext{
${ }^{1}$ Francisco García Marquina, Guía del viaje a la Alcarria, ed.cit.,p.200

${ }^{2}$ Sara Suárez, El léxico de Camilo José Cela, op.cit., p.59

${ }^{3}$ Ibíd., p.72, 73.
} 
y cuyos vocablos predominantes están polarizados en categorías semánticas de ruina, tristeza, nostalgia e inocencia, como sentarse al borde del camino, a hablar con la gente, acaba enviciando ${ }^{1}$.

Dentro de los ejemplos que podemos clasificar como vocabulario local y que son documentados en los libros que critican la obra de Cela generalmente y V. A en especial o por Cela mismo en sus dos novelas, citamos los siguientes ejemplos:

El viejo mendigo de Brihuega utiliza constantemente clichés de contenido religioso, habituales en un pueblo marcado profundamente por la fé católica: Ave María, Dios mediante, frescos nos dé Dios, será lo que Dios quiera...y la habitual de presentación: Rosario Gonzales, para servir a Dios y a usted ${ }^{2}$.

También son ilustrados los modos de locución coloquial, llena de egocentrismo, utilización de formas deícticas (alusiones a situaciones u objetos presentes o próximas a los hablantes), uso de muletillas y frases hechas, elementos suprasegmentales, acción física acompañante, etc ${ }^{3}$.

De ello hizo alarde el viejo Portillo en Brihuega y todo es clasificable como formas especiales de la gente del pueblo.

\footnotetext{
${ }^{1}$ Francisco García Marquina, Guía del viaje a la Alcarria, op.cit., p.204

${ }^{2}$ Ibíd., p. 200

3 Lorenzo, Emilio, Consideraciones sobre el lenguaje coloquial, en Comunicación y lenguaje, ed. Karpós, 1977, Madrid, p.165.
} 
Lo más importante que podemos anotar en las dos obras es las distinciones filológicas que hace Cela. El autor alude muchas veces al parador. Francisco García Marquina dice sobre los paradores en V. A:

En la Alcarria sólo había "posada" y "parador", [...] según Sebastián de Cobarrubias, gramático toledano de finales del siglo XVI, posada es la casa donde se reciban huéspedes que pasan su rato y su cansancio. En el mesón se ofrece albergue a las personas y a sus caballerías. Y la venta que presumo sinónimo de parador, sería una casa cercana al camino real para pasar el mediodía o la noche. [...] los paradores son caserones situados a las afueras donde se guarecen coches y carros y donde suelen dar mejor trato a los animales que a las personas ${ }^{1}$.

En la Alcarria, entonces, no saben la palabra mesón sólo saben parador, dice Cela mismo en su primer V. A:

- El viajero averiguó en este pueblo que en la Alcarria no conocen la palabra mesón. Preguntó por el mesón y ni le entendían. Fue cuando preguntó por la posada cuando le dijeron que posada no había, pero que sí había parador. P.84

El viajero afirma más tarde cuando visitó al pueblo de Casasana, que en la Alcarria no utilizan la palabra mesón, y al mismo tiempo nos da la diferencia en la significación que hay entre parador y posada:

\footnotetext{
${ }^{1}$ Francisco García Marquina, Guía del viaje a la Alcarria, op.cit., p.145. 
- El viajero, que ya había averiguado que mesón es una palabra desconocida en la Alcarria, aprende a distinguir entre parador y posada. El parador es una posada, con cuadra. En Casasana hay posada, pero no hay parador. P.125

Solamente en el pueblo Pareja dice el viajero, en vez de parador, fonda:

- El viajero hubiera sentado sus reales en Pareja, en la fonda de la plaza. P.117

Otra ocupación por la manera de habar de la gente de Alcarria fue mostrada a través de los comentarios sobre la pronunciación:

- A mi me dejaron en la mayor indingancia. El viajero tardó unos instantes en entender que había querido decir indigencia. P.80

En esta parte el viajero trata de mostrar como habla la gente pobre, en otra parte dice afirmando:

- El viajero empieza a pensar que el hombre de las cintas de colores tiene una dialéctica desconcentrada. P.80

Dentro del vocabulario que podemos llamar campesino, también se nota:

- ¡Tó, Generosa! ¡Arre, Generosa! P.45 
Podemos afirmar que Cela es aficionado por los comentarios filológicos, así también encontramos muestras en su N. V. A:

— Los señoritos de Madrid no distinguen un olmo de una olma, ¡allá ellos! La olma de Pareja tiene quinientos años, viene desde los reyes católicos y el descubrimiento de América, y es frondosa, noble y maternal, parece la imagen de la más próvida y saludable opulencia, y se enseña con una defensa de cemento todo alrededor. P.170

- A la entrada del pueblo hay una hermosa avenida de olmos y olmas. Los olmos son los que acaban en punta y las olmas son las que tienen un ramaje copudo, redondo, maternal. P.131

- El único habitante que se enseña es una niña con un lazo color salmón del tamaño de una paloma, que se centrillo con parsimonioso y muy rítmico deleite.

¿Por aquí le decís centrillo?

-Sí, señor, queda más claro que columpio. Pp. 187, 188

- El viajero escucha, medio confuso y distraído, quizás no haya apuntado con el debido orden, que algunos, a los de Alcocer, les llaman chileros; a lo mejor esto no es así y los chileros son los de Córcoles, como ya quedó dicho. P.190

- Millana fue de don Enrique de Villena el Nigromantico y a los naturales de aquí les dicen hidalgos. P.190

- Los muleros de Maranchón hablan entre si, en chalán, jerga que no se entiende demasiado. Los de Fuentelsaz y Milmarcos lo que hablan es la mingaña o magaña, enrevesada jerigonza que se comprende aún menos. P.191 
Cela cuando llegó por primera vez a la Alcarria:

Quiere espacios anchurosos y desconocidos para pintar una región abandonada. Camina con los ojos bien abiertos y un caudal de palabras olvidadas ${ }^{1}$.

Su segundo viaje especialmente, se considera como un retrato de usos de lengua y léxico alcarreño, donde abundan, como hemos visto a través de los ejemplos, las descripciones de este tipo:

La pureza del empleo del idioma, lo ha convertido en libro de texto en muchas universidades extranjeras y no sólo ha servido para el estudio del idioma castellano, sino también de la fisonomía de la España rural de la posguerra $^{2}$.

Según Cela mismo el libro de viaje es:

Un cuaderno para apuntar lo que en le diccionario no viene apuntado y, si alguna vez vino, fue borrado antes de tiempo ${ }^{3}$.

\subsection{Los tecnolectos}

Por tecnolecto entendemos la variedad lingüística que surge como código preciso de comunicación, generalmente entre especialistas, en relación con una materia determinada. Lo que se conoce normalmente como lenguaje

\footnotetext{
${ }^{1}$-Manuel Leguineche, Camilo José Cela, Vuelta a la Alcarria, El Cultural.ES, 17/01/2002.

2 - Francisco García Marquina, Guía del viaje a la Alcarria, op.cit., p.27

${ }^{3}$ Camilo José Cela, Judio, moros y cristianos, Barcelona, Destino, 1966, p.155
} 
especializado o específico, en oposición al lenguaje general o común, que comprende el saber lingüístico primario de un hablante. La noción de tecnolecto no debe ser confundida con la de sociolecto que como aludimos antes viene determinado por el grado de instrucción y conocimiento de la lengua (nivel sociocultural de la persona). Las diferencias vienen marcadas por el ambiente social y cultural de cada individuo, por sus estudios, etc. Mientras que los tecnolectos suelen generar de la utilización de términos específicos de actividades científicas, jurídicas, históricas, literarias, músicas, médicas, etc. Los tecnolectos pues, se refieren a unos rasgos funcionales:

Dice José Luís Fernández (2007) explicando las variedades funcionales en los dos viajes de Cela a la Alcarria:

Esto trae como consecuencia una obra de gran sencillez expresiva pero funcional, pues constantemente anda a la caza y captura del elemento que nos diga lo más que pueda con el menor número de palabras y sintagmas, y que lo haga con belleza y armonía literarias; una sencillez que está muy estudiada y que rinde cuentas a lo etnográfico, lo folclórico, lo sociológico, lo histórico o lo artístico de la España de posguerra ${ }^{1 .}$

De este tipo de variedades tecnolectos se abunda las dos obras de Cela, citamos a continuación unos tantos ejemplos que encontramos tan importantes en las dos obras:

Durante el viaje ha sido apuntado- en cuadernillos escolares, cartas de 
visita, sobres, fichas de cartón, hojas de papel antiguos, programas de televisión y hojas de revistas- impresiones, descripciones de paisajes, retratos, reflexiones personales sobre temas variados, precisiones geográficas o históricas, comentarios etimológicos y toponímicos, datos sobre los pueblos que recorre ${ }^{2}$.

En otra ocasión Carmen Aznar afirma (2004):

Por momentos adopta un tono pedagógico e introduce digresiones o "microensayos" sobre temas muy variados: literatura, filosofía, etimología, toponimia, geografía, hidrografia, topografia, geopolítica, historia, pintura, arquitectura, música, tradiciones, tauromaquia ${ }^{3}$.

En este caso hay que aludir que la profesión de cada hablante:

Influye de forma directa sobre la variación lingüística. [...] La función social de una persona, la actividad que realiza en una comunidad, está en relación directa con el lugar que ocupa en la jerarquía social y la valoración que de ella hacen los demás miembros de la comunidad ${ }^{1}$.

Así, pues surge como tecnolectos en las dos obras términos que aluden a los siguientes campos:

\footnotetext{
${ }^{1}$ José Luis Campal Fernández, Camilo José Cela, Viajero literario, Malabia: arte, cultura y sociedad, Barcelona, Montevideo, La Plata, Abril 2007.op.cit.

${ }^{2}$ Carmen Aznar Pastor, los libros de viaje de Camilo José Cela: ¿relatos autobiograficos o relatos de ficcion?, Quimera nro 246-247, Julio-agosto 2004

3 Ibídem.
} 


\subsubsection{Promoción comercial}

La promoción comercial se nota en la narración de Cela para describir detalladamente algunos oficios conocidos en la Alcarria:

- Barbería de La Puerta. Precio por cada servicio. Afeitado, 0,75. Corte, pelo raso, 0,75. ídem para atrás o raya, 1,00. Ídem a la parisién, 1,50. Servicio de brillantina, 0,25. Fricción colonia, 0,50. Señores corte de pelo, 1,00. La Puerta, $1^{\circ}$ de enero de 1945. El oficial Pablo Balcón. Servicio permanente de barbería de 11 mañana a 11 de la noche. Día en semana, jueves. V. A

\subsubsection{Lenguaje jurídico}

Aunque no aparece con frecuencia, sin embargo, queremos que es importante mencionar algún ejemplo que destaca esta variedad de lenguajes utilizados en las dos obras.

- El aplicar el raciocinio de las mentes que entendemos como normales, a las acciones de quienes nacen y viven y mueren en condiciones infrahumanas, es error en el que suelen caer, con frecuencia, los jueces y los historiadores. Contra el huero- o fantástico- principio de que todo hombre es igual ante la ley, proclamo que tal declaración no podrá entenderse como axiomática hasta que también sean iguales para todos los hombres las

${ }^{1}$ Francisco Moreno Fernández, Principios de sociolingüística y sociología del lenguaje, $1^{\mathrm{a}}$ 
posibilidades de sus condiciones de existencia, situación que entiendo todavía lejana. Si los Estados tuvieran la flexibilidad de que adolecen los jueces serían reclutados entre los psiquiatras (el mero tecnicismo jurídico es fácil de adquirir). P.124- V. A

\subsubsection{Historia}

Se puede deducir que la mayor parte los que hemos llamado como tecnolectos se encuentran dentro del campo historia tanto en V. A como en Nuevo viaje a la Alcarria.

- El viajero aprende que el castillo lo hizo don Juan Manuel y la iglesia una querida de Alfonso el Sabio que se llamaba doña Mayor. El viajero recuerda vagamente, que en un libro que leyó, hace años, llamaban a don Juan Manuel Turbulento y pendenciero. P.72 - V. A - En Castejón de Henares enterró el Cid los ricos caudales que conquistó a los moros y que, por más que se lleva escarbado, siguen sin aparecer; los indígenas se consuelan de su escasa fortuna zahorí jugándose los cuartos al guiñote, que es suerte de naipes semejante al tute. P.33 - N.V.A

\subsubsection{Filología}

Abundan en los dos viajes las descripciones filológicas usadas por Cela con el fin de explicar el uso de algunas palabras o dar significados a otras poco conocidas o bien porque son de uso campesino o bien

ed., ed. Ariel, S. A., Barcelona, 1998, p.60. 
porque vienen de otras lenguas extranjeras:

- Para mejor adoctrinamiento de ignorantes se aclara aquí lo que ya medio supone el diccionario: que el olmo crece derecho y que, cuando se capa, esto es, cuando se poda por la cruz y anchea, al olmo se le llama olma. P. 207- N.V.A

\subsubsection{Ciencia Geográfica}

Aparte de esto, hay que anotar las descripciones de ciencia geográfica que abundan en su primer viaje, nos quedamos, sin duda, ante un geólogo que se preocupa por dar explicaciones científicas de los fenómenos que se encuentran en su camino, como ejemplos citamos:

- En la parroquia del Salvador hay un púlpito de jaspe o de alabastro [...] tiene unas esculturas muy cuidadosamente esculpidas y lo remeta, por debajo, una cabeza con dos caras, como la de Jano, sólo que de hombre y mujer. El curo le cuenta al viajero la última historia del púlpito. P. 99

- Quico le explica al viajero que, según dicen, el monasterio de Óvila se lo llevaron los americanos, piedra a piedra, antes de la guerra civil. P.92

- Las dos Tetas son casi exactamente iguales vistas desde el norte, quizás la de poniente sea algo más alta. Tienen forma de cucurucho cortado antes de la punta y terminan, cada una, en una mesa de bordes rocosos y cortados a pico que deben ser difíciles de escalar. P. 80

- Al llegar a la vertiente, el viajero se encuentra ante una vista 
hermosa al principio y un poco desolada más allá. Del atajo empiezan a salir caminos, algunos casi borrados. P.95

Los libros de viajes se caracterizan por tener un discurso mixto, son textos que poseen un mensaje múltiple, Diana Calcines (1995) explica en su tesis doctoral sobre la literatura de viajes:

El hecho de que a un mismo tiempo sean textos narrativos y textos enciclopédico-descriptivos permite que con ellos se puedan coordinar materias como lengua y literatura, geografía, historia y ciencias naturales. Entre las muchas actividades que se pueden llevar a cabo a partir de un libro de viajes, se pueden destacar: el trabajo de un vocabulario específico, regional, gremial o científico. El comentario de los hechos históricos relatados por el autor, que suelen contener opinión; la localización de los lugares del itinerario de un mapa; comparación de las diferentes descripciones de los monumentos. ${ }^{1}$

En otra ocasión analiza también los libros de viaje de Camilo José Cela en especial con la siguiente manera:

Los libros de viaje de Camilo José Cela se caracterizan por poseer un discurso mixto. En ellos, el mensaje literario alterna con un discurso científico, rico y variado, que se halla entremezclado en el corpus literario de la narración del viaje [...]. Este discurso enciclopédico nunca aparecerá como una enumeración de datos, sino

${ }^{1}$ Diana Salcines De Delás, Literatura de viajes -una encrucijada de textos, Tesis doctoral, Universidad Complutense de Madrid, Dep. de filología Románica, Madrid, 1995, pp.167, 168. 
que a través de toda una serie de recursos literarios, diálogos inventados, etc. ${ }^{1}$.

No hay duda que dentro de las dos obras se abunda más el uso del diccionario geográfico e histórico debido a la naturaleza de los libros de viaje y al interés de Cela de reflejar todo detalle histórico o geográfico.

${ }^{1}$ Ibíd., p.167. 


\section{CONCLUSIONES}

En el presente estudio hemos intentado hacer un análisis del discurso mediante el estudio de los factores que más caracterizan los viajes escritos por Cela sobre la Alcarria. Del mencionado análisis se ha llegado a las siguientes conclusiones:

La mayoría de los personajes en las dos obras dependen en su discurso del uso de las comparaciones. Por ser la Alcarria una zona rural, donde el contacto con la naturaleza es continuo. Así surge una gran cantidad de comparaciones, en las cuales los términos iluminantes varían entre el reino animal, los seres humanos, objetos, plantas y naturaleza inanimada.

Tanto los personajes de la Alcarria como el autor de las dos obras son aficionados por el uso de los diminutivos. Los sufijos -ín, -ico, -uelo son los menos usados, mientras que -ito, illo son los más abundantes. Así, pues, Cela estaba muy interesado de reflejar el tono tradicional de la gente del campo. Lo hacía con la ayuda del uso de los diminutivos.

Es muy frecuente el uso del vocabulario coloquial especializado de la Alcarria y que se abunda, especialmente, entre los niños que aparecieron en los viajes. Asimismo se abundan el uso de los sustantivos y adjetivos de carácter coloquial que recoja Cela para designar o calificar a la conducta moral de los personajes.

Se nota que $90 \%$ de los personajes en las dos obras son gente vulgar y callejera y por lo tanto, abunda el uso de los vulgarismos. 
Se puede concluir que las personas que aparecieron en las dos obras usan lo que se llama unidades fraseológicas a fin de reforzar el sentido mediante los refranes y sentencias en forma pintoresca y a veces grotesca.

El análisis de las dos obras partió principalmente sobre dos pilares esenciales, primero el factor lingüístico antes mencionado y segundo el factor social, esto es, las diferencias entre los hablantes debidas al sexo, edad, nivel sociocultural y zona geográfica. Por ello se puede concluir lo siguiente partiendo de una perspectiva meramente social:

En la Alcarria de los cincuenta y de los ochenta cada uno tiene su apodo. El uso de los apodos es una característica muy clara en la Alcarria, la cual se reflejaba en las obras de Cela mediante los personajes de los dos viajes.

Cela en sus dos viajes a la Alcarria se concentró sobre la gente pobre. La vida del campo fue reflejada a través de la gente rural. Casi todos los personajes de las dos novelas son campesinos que utilizan, sobre todo, vocabulario aldeano. Así pues, se puede concluir una cantidad muy abundante del diccionario dialectal de la Alcarria como lo asegura Francisco García Marquina (2002):

Mientras el viajero reposa y charla con los vecinos podremos hacer una ligera excursión lingüística sobre el léxico que Cela empleará en su viaje a la Alcarria y que procede del alcarreo que ha realizado en el camino y cuyos vocablos predominantes están polarizados en categorías semánticas de ruina, tristeza, nostalgia e inocencia, como sentarse al borde del camino, a hablar 
con la gente, acaba enviciando ${ }^{1}$.

El uso de lenguajes especializados (los tecnolectos). Siendo los libros de viaje obras donde a un mismo tiempo se puede encontrar textos narrativos, enciclopédico-descriptivos permite que con ellos se puedan coordinar materias como lengua y literatura, geografía, historia y ciencias naturales. Lo cual sucede en las dos obras, en donde aparece la utilización de términos específicos de actividades científicas, jurídicas, históricas, literarias, músicas, médicas, etc.

En lo que respecta a la edad, hay que señalar que el empleo de las formulas de cortesía está favorecido por los individuos de más de 55 años y los niños de menos de 18 años. Además, se observa el uso de las interjecciones y locuciones interjectivas entre los habitantes de la Alcarria tal como ¡Caramba!, ¡Caray! ¡Huy! ¡Eh! ¡Jo! ¡Coño! ¡Psche! y ¡Zas! Todos son de uso frecuente en la Alcarria.

\footnotetext{
${ }^{1}$ Francisco García Marquina, Guía del viaje a la Alcarria, op.cit., p.204 


\section{Bibliografía}

Alvar, Manuel, Lengua y sociedad, Biblioteca cultural, Madrid, 1995.

, Manual de dialectología hispánica, Ed. Ariel S. A., Barcelona, 1999.

Amusategi, Karmele Rotaetxe, Sociolingüística, Ed. Sintesis, Madrid, 1990.

Briz, Antonio, El español coloquial: Situación y uso, Arco $\backslash$ Libros S.L., $2^{\mathrm{a}}$ ed., Madrid, 1998.

Carratalá, Fernando, Manual de ortografía española, Acentuación. Léxico y ortografía, Ed. Castilla S.A., Madrid, 1997.

Cortes, Manuel Muñoz, El español vulgar, Descripción de sus fenómenos y métodos de corrección, Ministerio de Educación Nacional, Madrid, 1958.

De Diego, Vicente García, Dialectología española, $3^{\text {a }}$ edición, Ed. Cultura Hispánica, Madrid, 1978.

De Vega, Manual y Cuetos Fernando, Psicolingüistica del español, Ed. Trotta S.A., Madrid, 1999, P.271.

Esquivel, Francisco Manuel, La lexicografía en las variedades no-estándar, Universidad de Jaén, $1^{\text {a }}$ edición, Jaén, 2001.

Fernández, Francisco Moreno, Principios de sociolingüistica y sociología del lenguaje, $1^{\mathrm{a}}$ edición, Ed. Ariel, S. A., Barcelona, 1998.

Frontín, José Luis Giménez, Camilo José Cela- Texto y Contexto, Montesinos, Barcelona, 1985.

Ilie, Paul, La novelística de Camilo José Cela, Gredos, Madrid, 1978. 
Julia, Santos; Casanova, Julián; Sabaté Josep María Sole I; Villarroya, Joan; Moreno, Francisco; Victimas de la guerra civil, Artes Graficas Huertas, S.A., Madrid, 1999.

López, Javier Medina, El anglicismo en el español actual, Arco \Libros, S.L., Madrid, 1996.

Lorenzo, Emilio, Comunicación y lenguaje, Ed. Karpós, Madrid, 1977.

Marcellesi, Jean Baptiste y Gardin, Bernard; Introducción a la sociolingüistica, Gredos, Madrid, 1974, P.28

Marquina, Francisco García, Guía del viaje a la Alcarria, Aache, Madrid, 1993.

Retrato de Camilo José Cela, Society of Spanish and spanish-American Studies, Universidad de Colorado at Boulder (U.S.A.) 2005.

Mekinassi, Ahmed, Léxico de las palabras españolas de origen árabe, Ed. Cremades, Tetauan, 1963.

Mirando, José Alberto, Usos Coloquiales del español, Publicaciones del colegio de España, 1ª edición, Salamanca, 1992.

Olano, Concepción Otaola, Análisis lingüistico del discurso, Ed. Académicas S.A., Madrid, 2006.

Ordóñez, Salvador Gutiérrez, La oración y sus funciones, Arco-Libros, S.L, Madrid, 1997.

Payne, Stanley G., El Régimen de Franco, Alianza, Madrid, 1987.

Suárez, Sara, El léxico de Camilo José Cela, Ed. Alfaguara, S.A., Barcelona, 1969.

Toledo, María Isabel, Variedad léxica y diferencias gramaticales en el uso coloquial: Una muestra, Actas IX, Universidad de Navarra, 1998. 
Torrego, Leonardo Gómez, El léxico en el español actual- Uso y norma, Arco-Libros, S.L, Madrid, 1998.

Tudela, Mariano, Cela, E.P.E.S.A., Madrid, 1970.

Valderrama, Manuel Ramiro, El énfasis de la prosa de Cela, Universidad de Valladolid, Salamanca, 1995.

Vicente, Alonso Zamora, Camilo José Cela, Acercamiento a un escritor, Gredos, D.L. Madrid, 1962.

Vila, Luís Blanco, Para leer a Camilo José Cela, colección clásicos del siglo XX, Palas Atenea, Madrid, 1990.

M. Alvar Ezquerra, La enseñanza del léxico y del uso del diccionario, Arco/Libros, Madrid, 2003. 